\title{
Suppression of Noise Propagation between Power and Ground Planes Using Ferrite-Plated EBG Structure
}

\author{
K. KONDO $^{\text {a) }}$, S. YOSHIDA ${ }^{\text {a) }}$, and Y. TOYOTA ${ }^{\text {b) }}$ \\ a) NEC TOKIN Corporation, 6-7-1 Koriyama, Taihaku-ku, Sendai, Miyagi, 982-8510 JAPAN
}

b) Okayama University, 3-1-1, Tsushima-naka Kita-ku Okayama, 700-8530 JAPAN

\begin{abstract}
To confirm the feasibility of suppressing electromagnetic interference (EMI) in the inner layers of multilayered printed wiring boards (PWBs) and sustaining power integrity characteristics, influence of inserting a ferrite-plated thin film between the power and ground planes on the transmission characteristics were evaluated using four types of test boards with the following two conditions: one is the solid test board with a plane copper foil or the EBG (Electromagnetic bandgap) test boards with a one-dimensional lattice-shaped EBG structure consisting of large and small squares $15 \mathrm{~mm} \times 15 \mathrm{~mm}$ and $1.5 \mathrm{~mm} \times 1.5 \mathrm{~mm}$ in size; the other is whether the 3 or 6 $\mu \mathrm{m}$-thick ferrite film was deposited or not. The multiple peaks of the transmission coefficient $\mathrm{S}_{21}$ due to parallel plate resonances were observed in the solid test board without ferrite film but the peaks were suppressed over the wide frequency range up to $6 \mathrm{GHz}$ by applying the ferrite film. The EBG test board with the ferrite film provided not only wideband attenuation owing to the ferrite film but also large attenuation in the EBG stopband. The multiple peaks of in the driving point impedance $Z_{11}$ due to the parallel plate resonances were also successfully suppressed by applying the ferrite film exhibiting favorable power integrity characteristics.
\end{abstract}

Key Words: electromagnetic band gap, ferrite thin film, parallel plate resonance, printed wiring board

\section{INTRODUCTION}

Electromagnetic interference (EMI) and radiated emissions with frequencies up to several GHz cause serious problems inside advanced digital electronic devices such as personal computers and cellular phones. Such electronic devices generally contain multilayered printed wiring boards (PWBs), which are commonly composed of inner power and ground layers besides inner and/or outer signal wiring layers. The noise radiation from such PWBs can be due to parallel plate resonances between the power and ground layers. Therefore, noise propagation between the inner layers that can cause the parallel plate resonances needs to be suppressed, but it is not effective to mount the existing noise suppression parts such as impedance components or composite noise suppression sheets (NSS) [1] on the outer signal wiring layers.

To suppress the radiation of the electromagnetic noise generated in the inner layers of PWBs, we investigated the noise suppression effect of a ferrite-plated film [2] deposited with a thickness of $3 \mu \mathrm{m}$ directly on the inner power and/or the ground layers of PWBs [3]. The radiated emissions from the PWBs with the ferrite-plated film were suppressed in the $\mathrm{GHz}$ range from 2 to $5 \mathrm{~dB}$. This is because high frequency noise current in the inner layer is attenuated mainly due to the large and wide band imaginary part of complex permeability ( $\left.\mu=\mu^{\prime}-j \mu^{\prime \prime}\right)$. However, the attenuation caused by the ferrite film was limited to a few $\mathrm{dB}$ because the ferrite film was deposited on the outer surfaces of the inner power and ground layers [3]. Hence, larger attenuation is expected when the ferrite film is inserted between the power and ground layers.

Electromagnetic band gap (EBG) structures, which exhibit band elimination characteristics, are also promising for suppressing noise propagation in the inner layers of PWBs. Since the planar type of the lattice-shaped EBG structure [4] is simple in shape, it can be easily installed onto the inner power and ground planes of PWBs. The lattice-shaped EBG structures exhibit much larger attenuation than that caused by the ferrite film, which is tens of dB. However, the stopband is not sufficiently wide because there are passbands where the noise current flows on both sides of the lower and higher frequency regions.

The purpose of this study is to confirm the feasibility of suppressing high-frequency electromagnetic-wave propagation in the inner layers of PWBs with the ferrite-plated film placed between the power and ground planes, enhancing the EMI suppression effect of the EBG structure with the ferrite-plated film. This is because the high-frequency electromagnetic wave that propagates through the inner layers of PWBs generally comes from simultaneous switching 
noise caused by switching at digital circuits. The point is not only suppressing noise propagation but also sustaining power integrity (PI) characteristics, which is a requirement for the power and ground layers of PWBs.

\section{FABRICATION OF TEST BOARDS}

Four types of test boards shown in Fig. 1 were fabricated in this study. Test boards A and C are the solid test board with a plane copper foil. Test boards B and D are the EBG test boards with a one-dimensional lattice-shaped EBG structure consisting of large and small squares $15 \mathrm{~mm} \times 15 \mathrm{~mm}$ and $1.5 \mathrm{~mm} \times 1.5 \mathrm{~mm}$ in size. For Test boards $\mathrm{C}$ and $\mathrm{D}$, the ferrite film of $3 \mu \mathrm{m}$-thick, which was with the same chemical composition and permeability spectra as those in the previous study [3], was inserted between the power and ground planes (not on the outer surfaces of the planes) as shown in Fig. 2. The test boards with the $6 \mu$ m-thick film, which might exhibit more significant noise suppression effects but need approximately two times longer deposition time, were also fabricated in this study.

\section{EMI SUPPRESSION AND POWER INTEGRITY CHARACTERISTICS OF TEST BOARDS}

In order to confirm the feasibility of suppressing high-frequency noise propagation in the inner layers of PWBs, S-parameters along the longitudinal direction of the test boards were measured using a vector network analyzer. For evaluating PI characteristics, the driving point impedance $\left(Z_{11}\right)$ was calculated from the measured S-parameters.

\section{A. Suppression of Noise Propagation $\left(S_{21}\right)$}

Fig. 3 shows the transmission coefficient $S_{21}$ to evaluate suppression effect of noise propagation. The multiple peaks due to parallel plate resonances observed in $S_{21}$ must be at least suppressed. In addition, sufficiently large attenuation beyond $20-30 \mathrm{~dB}$ is expected when a radio frequency section on the PWB must be isolated from digital noise. As for the solid test boards without the ferrite film, the multiple peaks due to parallel plate resonances are observed. With the ferrite film, the resonances are successfully suppressed in the wide frequency range up to $6 \mathrm{GHz}$. This is because noise propagation is suppressed with the $\mu$ " effect of the ferrite-plated film inserted between the power and ground planes. This is because noise propagation is suppressed with the ferrite-plated film inserted between the power and ground planes. The $6-\mu \mathrm{m}$-thick film exhibited more attenuation than the $3-\mu$ m-thick film. From the viewpoint of

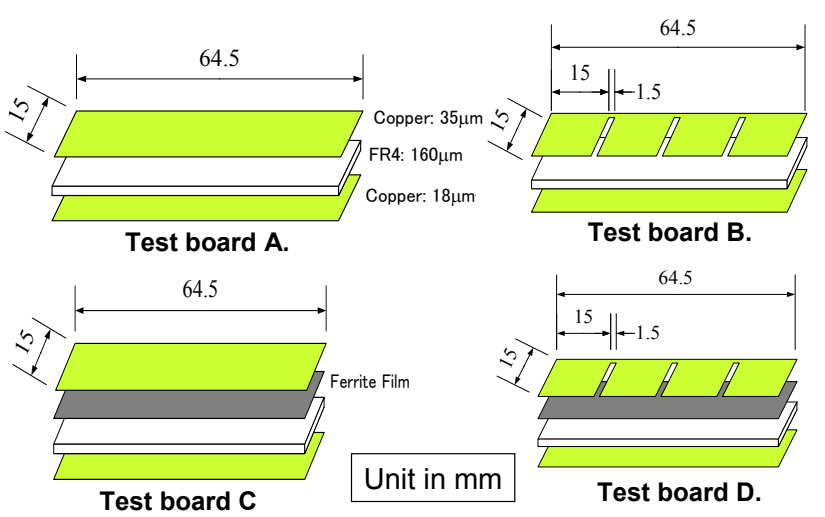

Fig. 1 Test boards fabricated in this study.

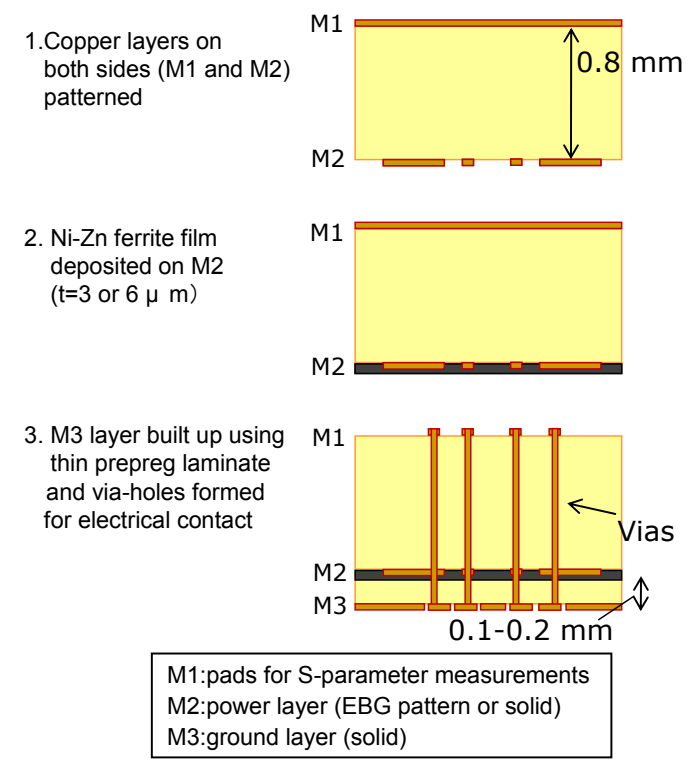

Fig. 2 Fabrication procedure of ferrite-plated test boards.

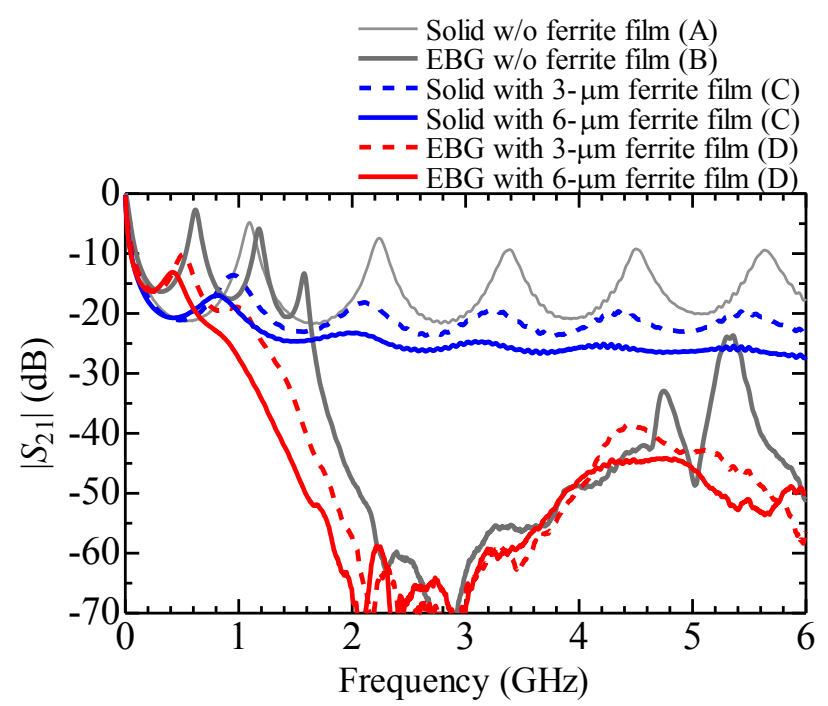

Fig. 3 Transmission coefficient $\mathrm{S}_{21}$ of test boards. 
fabrication process, however, the $3-\mu \mathrm{m}$-thick film seems to be more appropriate taking into account the longer deposition time needed for $6 \mu$ m-thick film.

The EBG test board without the ferrite film exhibits a clear stopband from 2 to $4 \mathrm{GHz}$ with the attenuation of 30 $\mathrm{dB}$ or more compared with solid test boards. The ferrite plated EBG structure provides a wider stopband than that of the EBG test board without the ferrite film. The frequency shift toward lower frequencies due to large $\mu$ ', approximately $0.3 \mathrm{GHz}$ and $0.7 \mathrm{GHz}$ for $3-\mu \mathrm{m}$-thick and 6 - $\mu \mathrm{m}$-thick films, causes much larger attenuation than those of the ferrite-plated solid test boards in the frequency range of 1-2 GHz because of the lower stopband edge. This

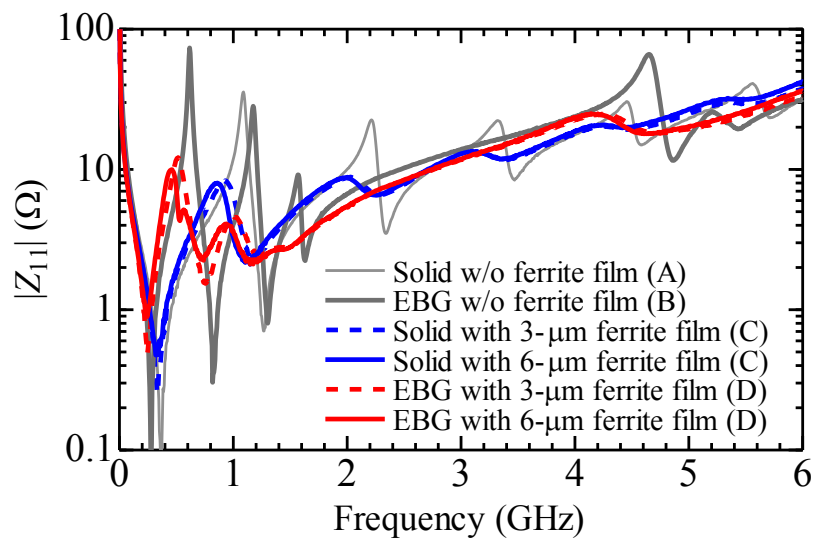

Fig. 4 Driving point impedance $Z_{11}$ of test boards. frequency shift indicates that the ferrite-plated film help miniaturize the EBG unit cell while

keeping the same band elimination characteristics within the thickness increment of several microns. In addition, it is also found that the ferrite-plated film contribute to remove the passband above $4.5 \mathrm{GHz}$, which corresponds to that of the EBG structure without the ferrite film.

\section{B. Power Integrity Characteristics $\left(Z_{11}\right)$}

Fig. 4 shows the $Z_{11}$ characteristics for evaluating PI characteristics. A lower $Z_{11}$ is the desirable requirements for PI characteristics of power/ground planes since the voltage fluctuation generated by the noise current at high

frequencies are reduced. At the passband frequencies of the EBG test board without the ferrite film, the multiple peaks of $Z_{11}$ are observed at frequencies of the peaks of $\mathrm{S}_{21}$, whereas the resonances were suppressed at the stopband frequencies.

For both of the solid and EBG test boards with the ferrite film, the $Z_{11}$ peaks due to the parallel plate resonances are suppressed in the $\mathrm{GHz}$ range. Around $0.5 \mathrm{GHz}$, however, the values of $Z_{11}$ for the EBG test boards with the ferrite film are beyond those of the solid test boards but the conventional electronic parts such as capacitors can easily reduce $Z_{I 1}$ at such lower frequencies.

As a result, it is found that the 3- $\mu$ m-thick ferrite film improves PI characteristics as well as the noise suppression characteristics of both the solid and EBG test boards. As for the 6 - $\mu \mathrm{m}$-thick film, the same tendency as the 3- $\mu \mathrm{m}$-thick film is obtained, suggesting that such a thick ferrite film should not be required.

\section{IV.CONCLUSION}

The ferrite film suppressed the noise propagations for the solid power/ground patterns and also enhanced the noise suppression effect for the EBG pattern. Furthermore, the ferrite-plated test boards exhibited favorable PI characteristics in spite of either EBG or solid pattern.

Considering all the results, we can conclude as follows. For the purpose of only reducing the parallel plate resonances, the insertion of the ferrite film between the power and ground planes is an adequate countermeasure. When sufficiently large attenuation is required, for example, when a radio frequency section on the PWB must be isolated from digital noise, the ferrite film combined with the EBG structure will be applicable.

\section{ACKNOWLEDGMENTS}

The authors thank Professor M. Abe, Professor N. Matsushita, and Dr. M. Tada at Tokyo Institute of Technology for very fruitful discussions. The authors also thank Dr. T. Watanabe at the Industrial Technology Center of Okayama Prefecture for his contribution to the fabrication of the test boards.

\section{REFERENCES}

[1] S. Yoshida, M. Sato, E. Sugawara, and Y. Shimada, J. Appl. Phys. 85, 4636 (1999). 
[2] M. Abe, M. Tada, N. Matsushita, and Y. Shimada, J. Appl. Phys. 99, 08M907 (2006).

[3] S. Yoshida, K. Kondo, H. Ono, and T. Kubodera, IEEE Trans. Magn. 44, 2982 (2008).

[4] J. Choi, V. Govind, and M. Swaminathan, Proc. IEEE Radio and Wireless Conf., Atlanta, GA, 2004, p.243. 